\title{
A First-Principles Study of a New Heusler Alloy
}

\author{
Chen Chen ${ }^{1}$, Jingbo Ren ${ }^{1}$, Zhenguo Wang ${ }^{1}$, Lei Feng ${ }^{1,2,{ }^{*}, \text { Wufeng Jiang }}{ }^{2}$, Suju Hao ${ }^{2}$ \\ ${ }^{1}$ North China University of Science and Technology Qian'an College, Tangshan, China \\ ${ }^{2}$ North China University of Science and Technology Metallurgy and Energy College, Tangshan, China
}

\author{
Email address: \\ feng_lei2000@163.com (Lei Feng) \\ ${ }^{*}$ Corresponding author
}

\section{To cite this article:}

Chen Chen, Jingbo Ren, Zhenguo Wang, Lei Feng, Wufeng Jiang, Suju Hao. A First-Principles Study of a New Heusler Alloy. International Journal of Materials Science and Applications. Vol. 6, No. 2, 2017, pp. 108-111. doi: 10.11648/j.ijmsa.20170602.17

Received: January 20, 2017; Accepted: February 22, 2017; Published: March 9, 2017

\begin{abstract}
A full-Heusler alloy $\mathrm{Ti}_{2} \mathrm{NiGa}$ have been investigated by first-principles calculations. The electronic structures and magnetic properties have been obtained. The compound is predicted to be a new half-metal ferrimagnet. The calculations show that there is an energy gap in the minority spin of the band structures, whereas the other spin is strongly metallic, which results in a complete spin polarization of the conduction electrons at the Fermi level. This is the obvious feature of a half-metal. The compound has a total magnetic moment of $3.0 \mu_{B}$ per unit cell on first-principles calculations which is in excellent agreement with the Slater-Pauling $(S P)$ rule. The magnetic moments of Ti(A) atom and Ti(B) atoms are different. This difference comes from different atom coordination surroundings of $\operatorname{Ti}(\mathrm{A})$ and $\operatorname{Ti}(\mathrm{B})$ atoms in crystal structure.
\end{abstract}

Keywords: Heusler Alloy, Half-Metal Ferromagnet, First-Principles, Band Structure

\section{Introduction}

With the rapid development of technology, various high-tech areas become increasingly demanding of new functional materials. There are a lot of functional materials have been found in the Heusler alloys. The Heusler alloys $\mathrm{Cu}_{2} \mathrm{MnAl}$ and $\mathrm{Cu}_{2} \mathrm{MnSn}$ were firstly found by $\mathrm{F}$. Heusler in 1903 [1], though there are no magnetic elements in the alloys, but they show magnetic characteristics. In 1969, a British P. Webster systematically studied the magnetic properties and crystal structure of the Heusler alloys for the first time [2]. Since then, thousands of similar types of intermetallic compounds have been found, these compounds are still in hot research, and new properties are increasingly being found by researchers. The half-metal, which maybe the most important spin injection source in spintronics have been found in Heusler alloys system. Since de Groot et al firstly introduced the concept "half-metal" in studying the half-Heusler alloy $\mathrm{NiMnSb}$ [3] in 1983, a lot of half-metal ferromagnets (HMFs) have been predicted in several different kinds of compounds: transition-metal oxides, such as $\mathrm{CrO}_{2}$ [4] and $\mathrm{Fe}_{3} \mathrm{O}_{4}$; perovskites, such as $\mathrm{Ca}_{2} \mathrm{FeMoO}_{6}, \mathrm{Sr}_{2} \mathrm{FeMoO}_{6}, \mathrm{Ca}_{2} \mathrm{FeReO}_{6}$; magnetically doped semiconductors such as Mn-doped GaN and GaAs; and the zinc-blended compounds such as $\mathrm{MnBi}$,
$\mathrm{CrSb}$, VTe. The so-called half-metal ferromagnet has the character that one spin direction is metallic at the Fermi level while the other is insulating (or semiconducting). The polarization is thus contributed entirely by one spin direction carriers at the Fermi energy. So it is of great interest to investigate HFMs from both theoretical and technical aspects. A lot of new HMFs have been found in Heusler alloy family. $\mathrm{Co}_{2} \mathrm{MnX}(\mathrm{X}=\mathrm{Si}, \mathrm{Ge})$ have been predicted to be HMFs by Ishida et al [5]. Later, $\mathrm{Mn}_{2} \mathrm{VAl}$ [6], $\mathrm{Co}_{2} \mathrm{CrAl}, \mathrm{Co}_{2} \mathrm{CrGa}$ and $\mathrm{Co}_{2} \mathrm{FeSi}$ [7-12] have also been reported to exhibit the characteristics of $\mathrm{HMFs}$ in their electronic structures. $\mathrm{Cr}_{2} \mathrm{MnAl}$ [13], $\mathrm{Cr}_{2} \mathrm{VX}(\mathrm{X}=\mathrm{Si}, \mathrm{Ge}, \mathrm{Sb})$ [14], $\mathrm{Cr}_{2} \mathrm{TiX}(\mathrm{X}=\mathrm{Ga}, \mathrm{Si}$, $\mathrm{Sn}, \mathrm{Sb})$ [15] have been reported to be or nearly be HMFs. Recently, $\mathrm{Ti}_{2} \mathrm{NiAl}$ [16], $\mathrm{Ti}_{2} \mathrm{FeGe}$ [17] also have been reported to be new HMFs. However, though atom $\mathrm{Ti}$ has similar electronic configuration as $\mathrm{Cr}$, Ti based Heusler alloy family has not been investigated well to explore for new HMFs.

In this paper, we have performed first-principles calculations on the electronic structure of Ti based full-Husler compound $\mathrm{Ti}_{2} \mathrm{NiGa}$ and aimed to seek a new HMF.

\section{Computational Details}

Many full-Heusler compounds have a cubic $\mathrm{L}_{21}$ structure. 
The structure is usually represented by the generic formula $\mathrm{X}_{2} \mathrm{YZ}$, where $\mathrm{X}$ and $\mathrm{Y}$ are transition metal elements and $\mathrm{Z}$ is a group III IV or $\mathrm{V}$ element. The structure can be seen as composed of four interpenetrating face-centered-cubic lattices with the crystal positions described with the Wyckoff coordinates are represented as A $(0,0,0), \mathrm{B}(1 / 4,1 / 4,1 / 4), \mathrm{C}$ $(1 / 2,1 / 2,1 / 2)$, and $\mathrm{D}(3 / 4,3 / 4,3 / 4)$. Among them, the $\mathrm{A}$ and $\mathrm{C}$ sites are similar in space surroundings. Usually, the $A, C$ and the $\mathrm{B}$ sites are occupied by the $\mathrm{X}$ and $\mathrm{Y}$ atoms and the $\mathrm{D}$ site is occupied by the $\mathrm{Z}$ atom. It is known that in a given Heusler alloy, even if the atom is same, the physical properties such as energy and magnetic moment are different when atoms enter different sites. It has been confirmed that atoms with more $3 \mathrm{~d}$ electrons prefer to occupy the $\mathrm{A}, \mathrm{C}$ sites and those with fewer tend to occupy $\mathrm{B}$ sites [18]. In $\mathrm{Ti}_{2} \mathrm{NiGa}$, because $\mathrm{Ni}$ atom has more $3 \mathrm{~d}$ electrons than $\mathrm{Ti}$ atom, $\mathrm{Ni}$ atoms prefer to enter site $\mathrm{C}$ $(1 / 2,1 / 2,1 / 2)$ while Ti atoms prefer to take sites A and B. As a result, a $\mathrm{Hg}_{2} \mathrm{CuTi}$-type $\mathrm{L}_{21}$ structure is developed. Ti atoms entering sites $\mathrm{A}$ and $\mathrm{B}$ are denoted as $\mathrm{Ti}(\mathrm{A})$ and $\mathrm{Ti}(\mathrm{B})$ respectively and $\mathrm{Ni}$ atoms entering site $\mathrm{C}$ are denoted as $\mathrm{Ni}(\mathrm{C})$ The calculations are applicable and employed for $\mathrm{Hg}_{2} \mathrm{CuTi}$-type $\mathrm{Ti}_{2} \mathrm{NiGa}$ in the following study. In one cell of the material, the four $\mathrm{Ga}$ atoms form a tetrahedron, and the $\mathrm{Ga}$ atoms occupy the four vertex. The crystal structure of the $\mathrm{Ti}_{2} \mathrm{NiGa}$ is showed in Figure 1. The spin-polarized electronic structures calculations are carried out by the plane-wave pseudo-potential method [19], which is based on density functional theory that describes the electron-electron interaction [20-22]. A generalized-gradient approximation (GGA) method proposed in 1996 by Perdew, Burke and Emzerhof and the PBE scheme [23] are chosen to dealing with the exchange-correlation potential. Ultrasoft pseudo-potential and a plane-wave cutoff energy of $280 \mathrm{eV}$ are used in all calculations. Special k-points generated with $6 \times 6 \times 6$ mesh parameters grid are employed in the irreducible Brillouin zone for all calculations. The calculations continue until the energy deviation is less than $10^{-6} \mathrm{eV} /$ atom.

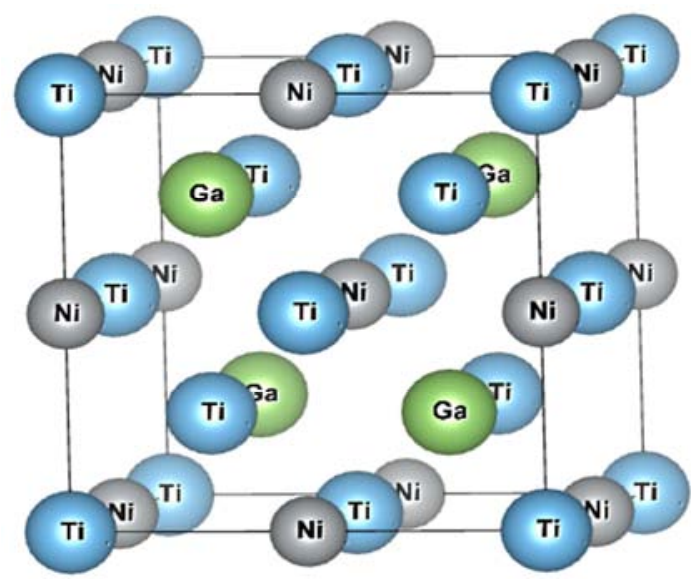

Figure 1. The unit cell of Ti2NiGa with Hg2CuTi-type crystal structure.

\section{Results and Discussions}

The geometry optimization of the crystal structure has been performed to determine the equilibrium lattice parameters. A diagram (Figure 2) of total energy versus lattice constant is obtained. The lowest total energy locates at $\mathrm{a}_{0}=6.18 \AA$ which is considered as the equilibrium lattice parameter. The following calculations are based on the $\mathrm{Hg}_{2} \mathrm{CuTi}$-type structure at $\mathrm{a}_{0}$.

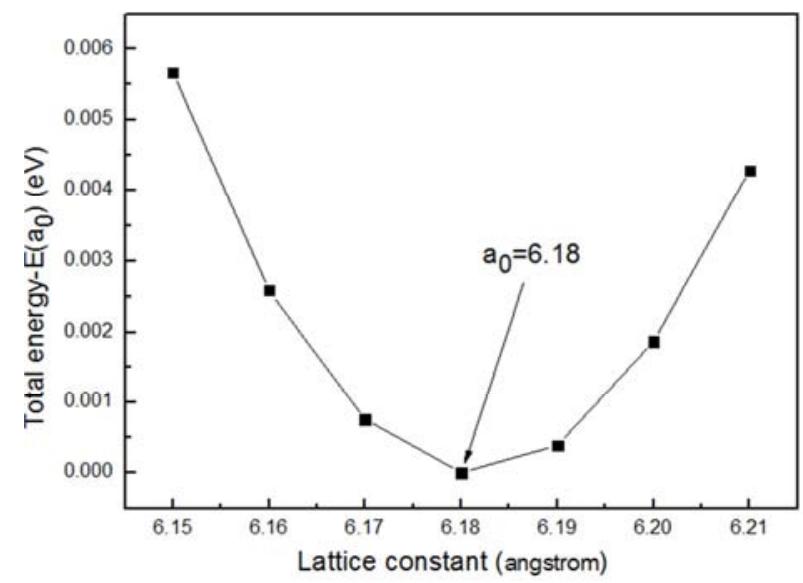

Figure 2. Calculated total energy vs lattice constant for Ti2NiGa full-Heusler compound with Hg2CuTi-type structure. The calculated equilibrium lattice constant is $6.18 \AA$ as indicated by arrow. The energy at equilibrium lattice constant $E(a 0)$ is chosen as zero in the vertical axis.

The calculated total moment of $\mathrm{Ti}_{2} \mathrm{NiGa}$ is precisely an integral value of $3.0 \mu_{\mathrm{B}}$ per unit cell at its equilibrium state, well consistent with the $S P$ rule $M_{t}=Z_{t}-18$ [24-26], where $M_{t}$ is the total moment and $Z_{t}$ is the total number of valence electrons per unit cell. The calculated total density of states (DOS) and atom-projected DOS (PDOS) are presented in Figure 3. For the total DOS, there is an energy gap about $0.75 \mathrm{eV}$ in the minority spin states around the Fermi level, demonstrating a half-metallic feature. The spin states of $\mathrm{Ni}(\mathrm{C})$ are mainly below the Fermi level and make a significant contribution to the total density of states. The magnetic moment of $\mathrm{Ni}(\mathrm{C})$ is $-0.06 \mu_{\mathrm{B}}$, which may be derived from its low exchange splitting in energy band. From the PDOS of Ti (A) and $\mathrm{Ti}(\mathrm{B})$, the minority spin states are shown to be mostly concentrated above the Fermi level. In the PDOS of Ti(A), the leading peak at $-0.52 \mathrm{eV}$ and $0.95 \mathrm{eV}$ in the spin-up and spin-down states make a large exchange splitting which leads to a large localized spin magnetic moment $1.96 \mu_{\mathrm{B}}$ of Ti(A) and to the polarization of $\operatorname{Ti}(\mathrm{A}) 3 \mathrm{~d}$ bands away from the Fermi level. In the PDOS of Ti(B), there are no obvious leading peaks for the up and down spin states and the densities are more dispersive on both spin directions which leads to a smaller spin magnetic moment of $\mathrm{Ti}(\mathrm{B})$ than that of $\mathrm{Ti}(\mathrm{A})$. The shapes of PDOS of Ti (A) and Ti (B) are different which is the similar case in $\mathrm{Cr}_{2} \mathrm{MnAl}$ [13]. In $\mathrm{Cr}_{2} \mathrm{MnAl}$, which is also a $\mathrm{HMF}$ with $\mathrm{Hg}_{2} \mathrm{CuTi}$-type full-Heusler structure, the shapes of PDOS of $\mathrm{Cr}(\mathrm{A}, \mathrm{C})$ and $\mathrm{Cr}(\mathrm{B})$ are very distinct. The differences of $\mathrm{Ti}(\mathrm{A})$ and $\mathrm{Ti}(\mathrm{B})$ in DOS shape should come from the atom coordination configuration [25] since $\mathrm{Ti}(\mathrm{B})$ atoms are located in a more symmetric coordination in contrast to $\mathrm{Ti}(\mathrm{A})$ atoms. The spin magnetic moment of $\mathrm{Ti}(\mathrm{B})$ is $1.36 \mu_{\mathrm{B}}$, smaller than 
that of $\operatorname{Ti}(\mathrm{A})$. The magnetic moment of atom $\mathrm{Ga}$ is $-0.26 \mu_{\mathrm{B}}$ which is unparallel with those of $\mathrm{Ti}(\mathrm{A})$ and $\mathrm{Ti}(\mathrm{B})$.

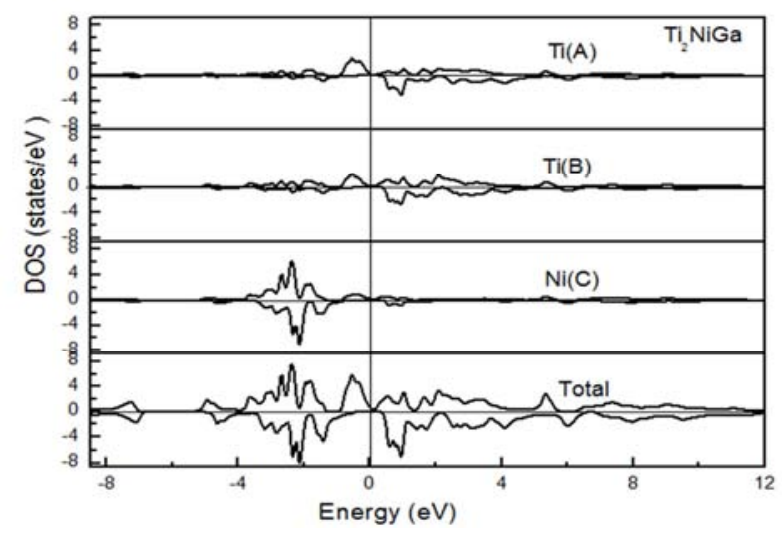

Figure 3. The calculated total DOS and PDOS for the $\mathrm{Hg}_{2} \mathrm{CuTi}$-type $\mathrm{Ti}_{2} \mathrm{NiGa}$ at the equilibrium lattice constant; the upper halves of each panel denote the spin-up states and the lower halves are the spin-down states.

The spin-dependent energy bands along high-symmetry directions in the Brillouin zone for $\mathrm{Ti}_{2} \mathrm{NiGa}$ are shown in Figure 4 . It can be seen that the majority spin-dependent band structure is strongly metallic, while the minority shows insulating character. It is obvious that there is an indirect band gap around the Fermi level in minority spin band. The Fermi level lies at $0.46 \mathrm{eV}$ above the minority spin valence band maximum (VBM) which is the minimum energy required to flip a minority spin electron from the VBM to the majority spin Fermi level [26]. The non-zero gap indicates that $\mathrm{Ti}_{2} \mathrm{NiGa}$ alloy is a typical half-metal.

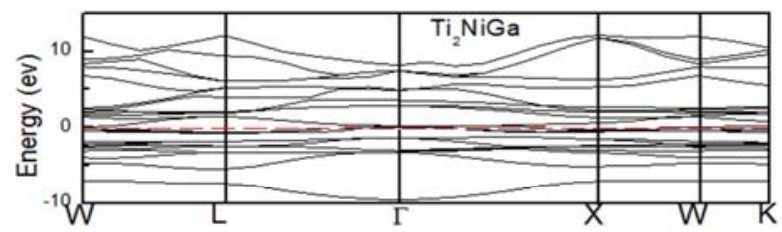

(a) majority spin

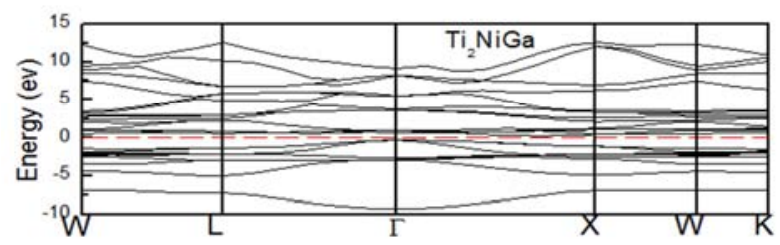

(b) minority spin

Figure 4. Majority spin (a) and minority spin (b) band structures for $\mathrm{Hg} 2 \mathrm{CuTi}$-type $\mathrm{Ti}_{2} \mathrm{NiGa}$ at the equilibrium lattice constant of $6.18 \mathrm{~A}$. The zero of energy denotes the position of the Fermi level.

In the paper, the crystal structure and the electric structure were studied using GGA method, but, one who had the experimental condition could try to prepare the alloy $\mathrm{Ti}_{2} \mathrm{NiGa}$, they would use X-ray diffraction method to confirm the structure, so the calculated X-ray diffraction was done, the data were plotted in the Figure 5. In the figure, the (111) diffraction peak is at 25 degree, and (200) diffraction peak is at 29 degree, the existence of the two diffraction peaks implies ordered crystal structure exists. Because the $\mathrm{Hg}_{2} \mathrm{CuTi}$-type structure is a high ordered crystal structure, the high order is sensitive to the impurities, so the metal raw materials should be high purity.

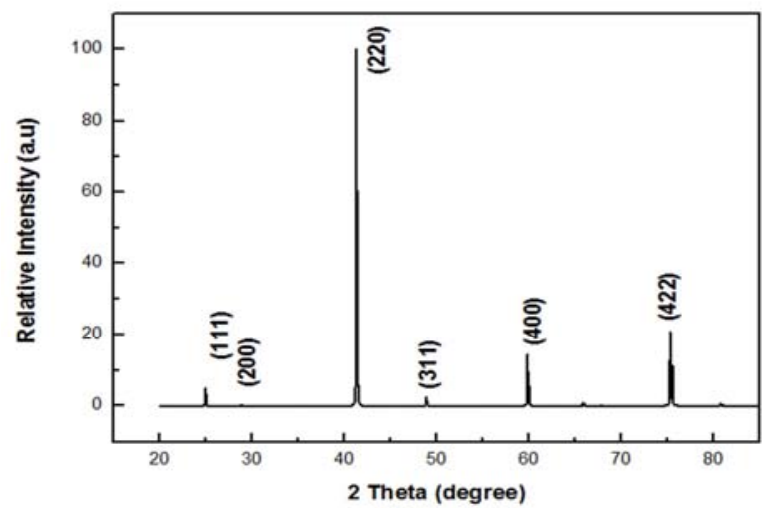

Figure 5. Calculated X-ray powder diffraction of $\mathrm{Ti}_{2} \mathrm{NiGa}$ with Hg2CuTi-type crystal structure.

\section{Conclusion}

In summary, $\mathrm{Ti}_{2} \mathrm{NiGa}$ with $\mathrm{Hg}_{2} \mathrm{CuTi}$-type structure is confirmed to be a new half-metal ferrimagnet on first-principles calculations and exhibits a promising potential for spintronics and other applications. It has a total moment of 3.0 $\mu_{\mathrm{B}}$, which is in excellent agreement with the SP rule. The band structures calculations also show the half-metallic character. The magnetic moments of $\mathrm{Ti}(\mathrm{A})$ and $\mathrm{Ti}(\mathrm{B})$ are $1.96 \mu_{\mathrm{B}}$ and $1.36 \mu_{\mathrm{B}}$ respectively which are larger that of $\mathrm{Ni}(\mathrm{C})$. The differences in magnetic moments between $\mathrm{Ti}(\mathrm{A})$ and $\mathrm{Ti}(\mathrm{B})$ should come from the different atom coordination configurations in crystal structure.

\section{Acknowledgements}

The authors acknowledge the support by the Department of Science and Technology of Hebei Province (grant No: 15211034), China and the Innovation Project of University Student of North China University of Science and Technology Qian'an College, Tangshan, China.

\section{References}

[1] F. Heusler, Verh. Dtscht. Phys. Ges. 5, 219 (1903).

[2] J. Pierre, R. V. Skolozdra, Yu. K. Gorelenko and M. Konacou, J. Magn. Magn. Mater. 134, 95 (1994).

[3] R. A. de Groot, F. M. Mueller, P. G. van Engen, and K. H. J. Buschow, Phys. Rev. Lett. 50, 2024 (1983).

[4] Lewis S. P, Allen P. B, and Sasaki T, Phys. Rev. B. 55, 10253 (1997).

[5] S. Ishida, T. Masaki, S. Fujii and S. Asano, Physica B. 245, 1 (1998).

[6] R. Weht and W. E. Pickett, Phys. Rev. B. 60, 13006 (1999). 
[7] A. Kellow, N. E. Fenineche, T. Grosdidier, H. Aourag and C. Coddet, J. Appl. Phys. 94, 3292 (2003).

[8] S. Ishida, S. Kawakami and S. Asano, Mater. Trans. JIM. 45, 1065 (2004).

[9] Y. Miura, K. Nagano and M. Shirai, Phys. Rev. B. 69, 144413 (2004).

[10] S. Ishida, S. Sugimura, S. Fujii and S. Asano: J. Phys.: Condens. Matter Vol. 3, (1991), p. 5793.

[11] R. Y. Umetsu, K. Kobayashi, R. Kainuma, A. Fujita, K. Fukamichi, K. Ishida and A. Sakuma, Appl. Phys. Lett. 85, 2011 (2004).

[12] S. Wurmehl, G. H. Fecher, H. C. Kandpal, V. Ksenofontov and C. Felser, Appl. Phys. Lett. 88, 032503 (2006).

[13] J. Li, Y. Li, G. Zhou, Y. Sun and C. Q. Sun, Appl. Phys. Lett. 94, 242502 (2009).

[14] J. Li, G. F. Chen, J. F. Li, Y. X. Li and C. Q. Sun, Scripta Materialia. 59, 1107 (2008).

[15] J. Li, G. F. Chen, H. Y. Liu, Y. X. Li, J. F. Li and X. W. Xu, J. Magn. Magn. Mater. 322, 1 (2010).

[16] Lei Feng, Chengchun Tang, Shuangjin Wang, Wenchen He, J. alloys and compounds. 509, 5187 (2011).
[17] F. Ahmadian, J. Supercond Nov Magn. 26, Issue 2, 381 (2013).

[18] H. C. Kandpal, G. H. Fecher and C. Felser, J. Phys. D. 40, 1507 (2007).

[19] S. Sanvito and N. A. Hill, Phys. Rev. B. 62, 15553 (2000).

[20] P. Hohenberg and W. Kohn, Phys. Rev. 136, B864 (1964).

[21] E. Wimmer, H. Krakauer, M. Weinert and A. J. Freeman, Phys. Rev. B. 24, 864 (1981).

[22] M. Weinert, E. Wimmer and A. J. Freeman, Phys. Rev. B. 26, 4571 (1982).

[23] J. P. Perdew, J. A. Chevary, S. H. Vosko, A. Jackson, M. R. Pederson and C. Fiolhais, Phys. Rev. B. 46, 6671 (1992).

[24] I. Galanakis, P. H. Dederichs and N. Papamkolaou, Phys. Rev. B. 66,174429 (2002).

[25] Kübler J, Physica B. 127, 257 (1984).

[26] Jung D, Koo HJ, Whangbo MH, J Mol Struct (Theochem). 527, $113(2000)$.

[27] J. Li, Y. Li, X. Dai and X. Xu, J. Magn. Magn. Mater. 321, 365 (2009).

[28] K. Capelle and G. Vignale, Phys. Rev. Lett. 86, 5546 (2001). 